\title{
Network-MIMO for downlink in-band relay transmissions
}

\author{
Sandra Lagen ${ }^{*}$, Adrian Agustin and Josep Vidal
}

\begin{abstract}
With the objective of improving the spectral efficiency and coverage homogeneity of wireless cellular systems in the downlink, we investigate how to take advantage of two promising transmission technologies envisioned in current standards: network-MIMO and relay stations (RSs). It is assumed that half-duplex RSs are deployed in a cellular system, where the duration of the relay-receive and the relay-transmit phases is fixed beforehand for all cells. In order to reduce the spectral efficiency loses associated to half-duplex relaying, we propose the use of base station (BS) cooperation under a network-MIMO precoding strategy based on block-diagonalization zero-forcing, and optimize radio resources under the convex performance criteria constrained by the per-BS power, modulation and coding schemes and the transmission rate in the relay-transmit phase. By applying convex optimization techniques, the optimal precoding strategy is derived and a suboptimal low complexity solution is also proposed. The obtained solutions are evaluated at system level and compared to other cooperative and non-cooperative BS-based schemes.
\end{abstract}

Keywords: Network-MIMO, Relay transmissions, Block-diagonalization zero-forcing, Convex optimization

\section{Introduction}

Due to the availability of new sophisticated terminals and bandwidth-demanding services, future cellular network designs are expected to provide high system spectral efficiency and homogeneous coverage. The design of such advanced network architectures is a challenging task, since radio resources like power and bandwidth are often scarce and the wireless channel presents unique impairments such as fading, multi-path, and interference. In this regard, next generation standards are already considering that conventional paradigms need to be rethought in a way able to exploit multi-antenna capacity increase, shadowing diversity, and path loss break concept. For that reason, mature enabling technologies, like multiple-input multiple-output (MIMO) or full frequency reuse planning, are an integral part of the system. However, more efficient wireless technologies and novel deployment concepts, like RS-based deployments or coordinated base station (BS) transmissions (often called network-MIMO or coordinated multipoint), are part of the ambitious study items [1] to deal with the

\footnotetext{
* Correspondence: sandra.lagen@upc.edu

Department of Signal Theory and Communications, Universitat Politecnica de Catalunya (UPC), Jordi-Girona 1-3,- Campus Nord, 08034, Barcelona, Spain
}

demanding traffic growth expected in the near future. Leveraging on the advantages offered by the joint use of all these techniques, to improve performance and cost efficiency, is a challenge faced by IEEE $802.16 \mathrm{~m} \mathrm{[2]} \mathrm{and}$ LTE-A [3].

One way to increase network capacity is by means of increasing the radio access points and reducing the cell size. However, since a regular BS is expensive, this solution is not attractive to network operators. Pursuing the same objective with lower cost, relay-based deployments is a promising approach that will have a large effect on system's functions and architectures [4,5]. While implementation details of full-duplex RS are still under investigation, relay-based enhancements in standards consider half-duplex relay operation [5], which incur a rate penalty as they require at least two timeslots to relay a message from source to destination [6,7]. It is therefore crucial to enhance the capacity of the in-band wireless backhaul between source and relay (in our case, the BS-RS link) to increase the information rate. One of the solutions usually assumed is that relay stations (RSs) are placed in specifically planned positions above rooftop or in lampposts, ensuring line-of-sight (LOS) conditions in the BS-RS link, and hence reducing the path

\section{穴}


loss and shadowing effects. However, the price to pay is twofold: the likely LOS propagating conditions also to other-cell BSs (which will inject harmful interference) and the rank deficiency of the spatial channel when both BS and RS are equipped with multiple antennas. Both effects are detrimental to MIMO channel gains $[8,9]$.

In this respect, network-MIMO [10] seems especially suited to address in-band backhauling in relay transmissions for the downlink. While coordination may be seen as an efficient way to combat the interference from neighbor cells [11,12], it also creates a virtual MIMO broadcast channel whose number of degrees of freedom is boosted (if compared to a conventional single-user MIMO under TDMA) and is hardly affected by the rank deficiency of single-user MIMO channels in LOS. It has been observed that network-MIMO based on blockdiagonalization zero-forcing (BD-ZF) performs closely to dirty-paper coding [10] but, although its simplicity, it requires accurate channel knowledge from all involved links. However, network-MIMO is again appropriate for our problem, thanks to the long channel coherence time of BS-RS links.

An additional way to improve the efficiency of relay transmissions is by optimizing the duration of the relayreceive and the relay-transmit phases [6,7]. In [13], we observed that the joint optimization of coordinated BS-RS links (through network-MIMO) and transmit duration phases brings large benefits. However, this approach is not convenient when considering multiple coordinated cells: if each group of coordinated cells adapts the duration of the transmission independently, the interference power observed in each transmission slot may be time-varying, a harsh, and undesirable situation for the cellular system. Therefore, so as to guarantee the predictability of other cell-clusters interference within transmission frames, the transmit duration phases should be fixed beforehand based on some criterion. For example, in [14], a statistical criterion was used and it was observed that still large gains both in terms of spectral efficiency and outage rate could be obtained.

Following the above motivations, we focus our study in the development of precoding strategies when network-MIMO based on BD-ZF is applied to the first hop of a relay-based transmission and the duration of the relay-receive and relay-transmit phases is fixed according to statistical approach. In this regard, we derive the optimal precoding strategy and compare it with other suboptimal and simpler solutions. The optimal precoding strategy is obtained in order to maximize the transmission rates subject to per-BS power constraints, modulation and coding schemes (MCS) constraints and maximum bitrates on the RS-UEs link, following similar steps used in [12] to obtain the optimal precoding for network-MIMO. On the other side, the suboptimal precoding strategy comes out by maximizing the transmission rates subject to a sum-power constraint [15] and after adapting the power allocated to each symbol stream so as to satisfy the per-BS power constraints, as it was done in [14]. As a result, the optimal precoder turns into a complex structure and the questions arising are

- How much a relay-based system can benefit in terms of cellular spectral efficiency and cell-edge spectral efficiency by BS cooperation?

- How much gain can be achieved with the optimal MIMO symbol precoding compared to suboptimal and simpler precoding solutions?

This article is structured as follows. In Section 2, we state the system model. The resource allocation problem is formulated in Section 3, in which we derive the optimal precoding strategy and also a suboptimal lowcomplex precoding strategy is proposed. In Section 4, simulation results are presented. Conclusions are drawn in Section 5.

Notation: For a given matrix $\mathbf{A}$, its transpose matrix is denoted as $\mathbf{A}^{\mathrm{T}}$ and its transpose conjugate matrix as $\mathbf{A}^{\mathrm{H}}$; $|\mathbf{A}|$ operator is referred to the determinant of $\mathbf{A}, \operatorname{tr}(\mathbf{A})$ to the trace, $\operatorname{rank}(\mathbf{A})$ to the rank (which is the minimum between the column and the row rank) and $\operatorname{kernel}(\mathbf{A})$ is denoting the null space of the matrix; righteigenvec(A) denotes a matrix containing the right eigenvectors of $\mathbf{A}$ and eigenval(A) denotes a matrix with the eigenvalues of $\mathbf{A}$ in its diagonal; $\mathbf{A} \geq \mathbf{0}$ means that matrix $\mathbf{A}$ is positive semi-definite; $\mathbf{A}=\operatorname{diag}\left(a_{1}, \ldots, a_{N}\right)$ describes a diagonal matrix with diagonal entries $a_{1}, \ldots, a_{N}$. For given scalars $a$ and $b$, min $(a, b)$ denotes the minimum between $a$ and $b$, and $(a)^{+}$denotes the maximum between $a$ and 0 .

\section{System model}

This section presents the model for a relayed-based deployment with network-MIMO applied to the in-band backhaul. In Section 2.1, some necessary system assumptions are detailed. Signal model in BS-RS link and signal model in RS-UE link are devoted to Sections 2.2 and 2.3 , respectively.

\subsection{System assumptions}

Our system definition is based on the following practical assumptions:

1. The number of antennas at BS, RS, and UE is $n_{\mathrm{B}}$, $n_{\mathrm{R}}$, and $n_{\mathrm{M}}$, respectively.

2. The quality of the backhaul link supports the exchange of perfect channel state information at the transmitter side (CSIT) of the first hop among the cooperating BSs. CSIT is assumed for transmissions from BSs to 
RSs since long time coherence is expected in those links as both terminals have static positions.

3. A maximum possible MCS is assumed.

4. RSs are time half-duplexed terminals operating under decode-and-forward. That is a suitable coding approach for BS-RS links, where high signal-to-noise ratio (SNR) is expected if LOS propagation is met [16].

5. User equipments (UEs) do not process the signals transmitted by the BS, only those transmitted by the RS. In other words, simple forwarding relaying is assumed.

6. No CSIT is available at RSs. RS transmissions are not coordinated in the way BS transmissions are. Their transmissions are interfered as multiple RSs transmit simultaneously (see Figure 1).

7. Each RS transmits to a single associated UE and therefore it is considered as a single user MIMO link with interference. Full CSIT may be exploited at the RS if sufficient feedback rate from the UE is allowed. Otherwise, only average CSIT is assumed.

8. It is assumed that the duration of slots for the BSRS links and for the RS-UE links is constant to preserve interference stationarity between cell clusters.
9. The scheduler implements a round-robin TDMA algorithm to serve users in groups of $R$ users.

10. The radio resource allocation (transmitted power, beamformers) is performed by prioritizing each UE selected by the scheduler, so that a certain quality of service (QoS) is provided. A reasonable criterion is that user's priority is taken as the inverse of the capacity in the RS-UE link so as to avoid unfair service to deprived users. This way, in comparison with the sum-rate (SR) criterion (equal priorities to all users), a weighted SR (WSR) criterion would perform better in terms of outage rate while losing in terms of spectral efficiency.

\subsection{Signal model in BS-RS link}

We shall adopt a downlink transmission setup where $B$ BSs are coordinated and assisted by $R$ RSs to transmit messages to $R$ UEs. Each UE is associated to a single RS. All BSs transmit on a fixed fraction of time $\alpha_{1}$ on the first hop to the RSs following a network-MIMO strategy based on BD-ZF [17] (see Figure 1), which is appropriate for BS-RS links in LOS conditions (MMSE precoding provides improved performance at medium to low SNR [18]).

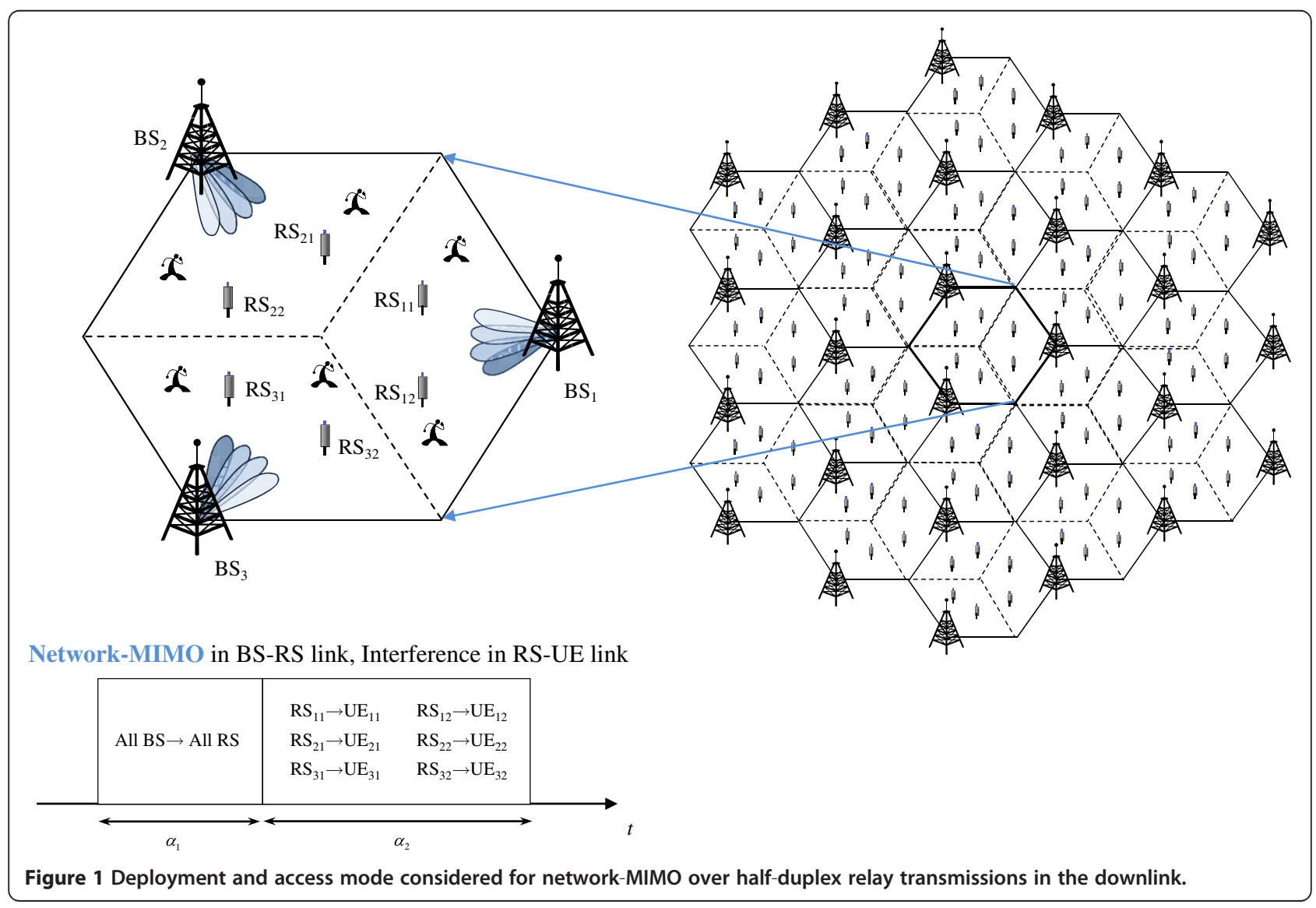


The signal $\mathbf{x}$ transmitted by all $n_{\mathrm{B}} \cdot B$ antennas is given by

$$
\mathbf{x}=\left[\begin{array}{c}
\mathbf{x}_{1} \\
\vdots \\
\mathbf{x}_{k} \\
\vdots \\
\mathbf{x}_{B}
\end{array}\right]=\sum_{i=1}^{R} \mathbf{Q}_{i} \mathbf{b}_{i}
$$

where $\mathbf{x}_{k}$ is the signal transmitted by the $k$ th BS, $\mathbf{b}_{i}$ is the symbol stream vector of $m_{i}$ independent components associated to the $i$ th $\mathrm{RS}$ and $\mathbf{Q}_{i}$ is its associated precoding matrix. We adopt a BD-ZF precoding [17] defined by two matrices

$$
\mathbf{Q}_{i}=\mathbf{V}_{i} \mathbf{S}_{i}
$$

where $\mathbf{S}_{i}$ is the $\left(B \cdot n_{\mathrm{B}}-(R-1) \cdot n_{\mathrm{R}}\right) \times m_{i}$ MIMO symbol precoding matrix, and $\mathbf{V}_{i}$ is the $B \cdot n_{\mathrm{B}} \times\left(B \cdot n_{\mathrm{B}}-(R-1)\right.$.

$\left.n_{\mathrm{R}}\right)$ BD-ZF precoding matrix. By virtue of the $\mathrm{ZF}$ precoding, the signal received by the $i$ th $\mathrm{RS}$ is affected by the $n_{\mathrm{R}} \times B \cdot n_{\mathrm{B}}$ channel matrix $\mathbf{H}_{i}$ (containing the channel gains between the transmitting antennas at the $B$ BSs and its receiving antennas):

$$
\mathbf{y}_{i}=\mathbf{H}_{i}\left(\mathbf{V}_{i} \mathbf{S}_{i} \mathbf{b}_{i}+\sum_{j=1, i \neq j}^{R} \mathbf{V}_{j} \mathbf{S}_{j} \mathbf{b}_{j}\right)+\mathbf{n}_{i} \in \quad i=1, \ldots, R
$$

where $\mathbf{n}_{i}$ is the additive complex white Gaussian noise.

The BD-ZF precoder design implies $\mathbf{V}_{i} \in \operatorname{kernel}\left(\widetilde{\mathbf{H}}_{i}\right)$, where

$$
\widetilde{\mathbf{H}}_{i}=\left[\begin{array}{llllll}
\mathbf{H}_{1}^{T} & \cdots & \mathbf{H}_{i-1}^{T} & \mathbf{H}_{i+1}^{T} & \cdots & \mathbf{H}_{R}^{T}
\end{array}\right]^{T}
$$

The existence of a kernel of appropriate rank requires $B \cdot n_{\mathrm{B}}>(R-1) \cdot n_{\mathrm{R}}$ and hence

$$
\operatorname{rank}\left(\mathbf{H}_{i} \mathbf{V}_{i}\right) \leq \min \left(n_{R},\left(B \cdot n_{B}-(R-1) \cdot n_{R}\right)^{+}\right)
$$

In addition, symbol decidability at the receivers implies the following conditions on the number of streams

$$
\sum_{m_{i} \leq \operatorname{rank}\left(\mathbf{H}_{i} \mathbf{V}_{i}\right) \quad i=1, \ldots, R}^{R} m_{i} \leq B \cdot n_{B}
$$

It must be remarked that in the case the $i$ th RS observes all coordinated BSs in LOS (hence BS-RS link channels are rank deficient) the rank of $\mathbf{H}_{i}$ grows up to full-rows rank provided that channels to the $B$ BSs be linearly independent.
Regarding matrix $\mathbf{S}_{i}$, it is designed to maximize users' transmission rates:

$$
r_{1 i}=\log _{2}\left|\mathbf{I}+\mathbf{N}_{i}^{-1 / 2} \mathbf{H}_{i} \mathbf{V}_{i} \mathbf{S}_{i} \mathbf{S}_{i}^{H} \mathbf{V}_{i}^{H} \mathbf{H}_{i}^{H} \mathbf{N}_{i}^{-H / 2}\right|
$$

where $\mathbf{N}_{i}$ refers to the correlation matrix of the noise $\mathbf{n}_{i}$ plus external interference at the $i$ th RS. In addition, we consider practical MCS in the BS-resource allocation, so we have to upper limit the rate for each transmission mode of each user on the BS-RS link

$$
r_{1 i} \leq S_{\max } m_{i} \quad i=1, \ldots, R
$$

where $S_{\max }$ is the maximum spectral efficiency that can be achieved by the LTE-A MCS [3] for each transmission mode.

The total power transmitted by the $k$ th $\mathrm{BS}$ is given by

$$
P_{k}=\operatorname{tr}\left(E\left\{\mathbf{x}_{k} \mathbf{x}_{k}^{H}\right\}\right)=\operatorname{tr}\left(\sum_{i=1}^{R} \mathbf{B}_{k} \mathbf{V}_{i} \mathbf{S}_{i} \mathbf{S}_{i}^{H} \mathbf{V}_{i}^{H}\right) \quad k=1, \ldots, B
$$

where $\mathbf{x}_{k}$ is the signal transmitted by the $k$ th BS (from Equation 1) and $\mathbf{B}_{k}$ selects the $n_{\mathrm{B}}$ rows of $\mathbf{V}_{i} \mathbf{S}_{i}$ used to transmit from the $k$ th $\mathrm{BS}$

$$
\mathbf{B}_{k}=\operatorname{diag}(\underbrace{0, \ldots, 0}_{(k-1) \cdot n_{B}} \underbrace{1, \ldots, 1}_{n_{B}} \underbrace{0, \ldots, 0}_{(B-k) \cdot n_{B}})
$$

\subsection{Signal model in RS-UE link}

On the second hop, each RS transmits to its associated UE on a fixed fraction of time $\alpha_{2}=1-\alpha_{1}$ (see Figure 1). Since $R$ relays can transmit simultaneously, interference is created. We could also split the second hop in different time slots, reducing interference at the expenses of some loss in spectral efficiency [13].

As we are assuming no coordination among RSs and simple receivers at the UE, only single user MIMO transmissions can be appointed. Then, the achievable rate for each RS-UE link, $r_{2}$, follows the conventional MIMO capacity expression affected by the presence of interference from other RS transmissions. If no CSIT is assumed at the RS

$$
r_{2 i}=\log _{2}\left|\mathbf{I}+\frac{P_{i}^{R}}{n_{R}} \mathbf{H}_{i i}^{H} \mathbf{H}_{i i}\left(\mathbf{N}_{i}+\sum_{j=1, j \neq i}^{R} \frac{P_{j}^{R}}{n_{R}} \mathbf{H}_{j i}^{H} \mathbf{H}_{j i}\right)^{-1}\right| i=1, \ldots, R
$$

where $r_{2 i}$ denotes the rate in the $i$ th RS-UE link, $P_{i}^{R}$ is the power transmitted by the $i$ th RS to its UE, and $P_{j}^{R}$ defines the power transmitted by the $j$ th RS. 
To preserve information flow through RSs, the rate at the $i$ th UE served by the $i$ th RS is constrained by the minimum of rates in both hops

$$
r_{i} \leq \min \left(\alpha_{1} r_{1 i}, \alpha_{2} r_{2 i}\right) \quad i=1, \ldots, R
$$

where $r_{1 i}$ is transmission rate on the BS-RS link for the $i$ th RS (6). Since the transmission time allocation is fixed and the transmission rate on the second hop $r_{2 i}$ is given by the propagation channel conditions in the RS-UE links, we should allocate power in the BS-RS link accordingly so as not to waste it. Then, Equation (11) can also be written as a constraint on the resource allocation in the BS-RS link

$$
r_{1 i} \leq \alpha_{2} r_{2 i} / \alpha_{1} \quad i=1, \ldots, R
$$

\section{Resource allocation}

This section is devoted to find the optimal power allocation and beamforming vectors for BS-RS links (both included in the MIMO symbol precoder $\mathbf{S}_{i}$ ) in order to maximize the WSR of the system, when there are per-BS power constraints and maximum MCS. Following similar steps as in [12] we obtain the optimal solution in WSR-based optimal resource allocation. Similarly, in WSR-based suboptimal resource allocation, a suboptimal and simpler solution for $\mathbf{S}_{i}$ is presented, which assumes the conventional structure for the beamforming in network-MIMO, [15], and then the power is allocated so as to satisfy the power and rate constraints [14].

\subsection{WSR-based optimal resource allocation}

We want to maximize users' transmission rates subject to per-BS power constraint to obtain the optimal MIMO symbol precoding matrix structure for matrices $\mathbf{S}_{i}$ in (2). If we consider $\mathbf{T}_{i}=\mathbf{S}_{i} \mathbf{S}_{i}^{H}$, the WSR maximization problem for the downlink transmission in relayed networkMIMO with BD-ZF precoding and per-BS power constraints is formulated as follows

$$
\begin{aligned}
&\left(P_{1}\right): \underset{\left\{\mathbf{T}_{i}\right\},\left\{r_{i i}\right\}}{\operatorname{minimize}}-\sum_{i=1}^{R} \mu_{i} r_{1 i} \\
& \text { s.t. } \begin{cases}\operatorname{tr}\left(\sum_{i=1}^{R} \mathbf{B}_{k} \mathbf{V}_{i} \mathbf{T}_{i} \mathbf{V}_{i}^{H}\right)-P_{k}^{\max } \leq 0 \quad k=1, \ldots, B \\
r_{1 i}-\log _{2}\left|\mathbf{I}+\mathbf{N}_{i}^{-1 / 2} \mathbf{H}_{i} \mathbf{V}_{i} \mathbf{T}_{i} \mathbf{V}_{i}^{H} \mathbf{H}_{i}^{H} \mathbf{N}_{i}^{-H / 2}\right| \leq 0 \quad i=1, \ldots, R \\
r_{1 i}-\min \left(\alpha_{2} r_{2 i} / \alpha_{1}, S_{\max } m_{i}\right) \leq 0 \quad i=1, \ldots, R \\
-r_{1 i} \leq 0 \quad i=1, \ldots, R \\
\mathbf{T}_{i} \geq \mathbf{0} \quad i=1, \ldots, R\end{cases}
\end{aligned}
$$

where $r_{1 i}$ is the transmission rate in the BS-RS link for the $i$ th UE and $\mu_{i}$ the priority given to it in order to add some QoS over the served users. The first constraint accounts for the per-BS power constraint (taken from (8)), where $P_{k}^{\max }$ denotes the per-BS available power at the $k$ th BS. The second constraint refers to the per-user transmission rate in the BS-RS link. The third constraint contains the peruser transmission rate, which is limited by the per-user transmission rate in the RS-UE link (from Equation 12) and the per-user MCS constraint in the BS-RS link (from Equation 7). Finally, the fourth constraint imposes that the transmission rates have to be positive, and the fifth constraint imposes that the transmit covariance matrices have to be positive and semi-definite.

With the inclusion of the new variable $r_{1 i}$ in the problem, it can be verified that problem $\left(P_{1}\right)$ turns out to be a convex optimization problem (as the objective function is convex over $\left\{r_{1 i}\right\}$ and all the constraints specify a convex set over $\mathbf{T}_{i}$ 's and $\left\{r_{1 i}\right\}$ [19]). Then, problem $\left(P_{1}\right)$ can be solved using standard convex optimization techniques, e.g., interior-point method [19]. However, the optimal structure for $\mathbf{T}_{i}$ and $r_{1 i}$ can be found in a closed-form using Lagrange duality methods $[19,20]$.

\section{Proposition 1}

Denoting by $\left\{\gamma_{k}\right\}$ and $\left\{\varphi_{i}\right\}$ a set of non-negative dual variables associated to the $k$ th per-BS power constraint and the $i$ th per-user transmission rate, respectively, the optimal solution to problem $\left(P_{1}\right)$ for a set of fixed $\left\{\gamma_{k}\right\}$ and $\left\{\varphi_{i}\right\}$ is given by

$$
\begin{aligned}
& \mathbf{T}_{i}^{*}=\left(\mathbf{V}_{i}^{H} \mathbf{B}_{\gamma} \mathbf{V}_{i}\right)^{-1 / 2} \mathbf{W}_{i} \mathbf{P}_{i} \mathbf{W}_{i}^{H}\left(\mathbf{V}_{i}^{H} \mathbf{B}_{\gamma} \mathbf{V}_{i}\right)^{-1 / 2} i=1, \ldots, R \\
& \mathbf{B}_{\gamma}=\sum_{k=1}^{B} \gamma_{k} \mathbf{B}_{k} \\
& \overline{\mathbf{H}}_{i}=\mathbf{N}_{i}^{-1 / 2} \mathbf{H}_{i} \mathbf{V}_{i}\left(\mathbf{V}_{i}^{H} \mathbf{B}_{\gamma} \mathbf{V}_{i}\right)^{-1 / 2} \\
& \mathbf{W}_{i}=\operatorname{righteigenvec}\left(\overline{\mathbf{H}}_{i}\right) \\
& \boldsymbol{\Lambda}_{i}=\operatorname{eigenval}\left(\overline{\mathbf{H}}_{i}\right)=\operatorname{diag}\left(\lambda_{i 1} \cdots \lambda_{i j} \cdots \lambda_{i m_{i}}\right) \\
& \mathbf{P}_{i}=\operatorname{diag}\left(p_{i 1} \cdots p_{i j} \cdots p_{i m_{i}}\right)
\end{aligned}
$$

Therefore, the power allocation and attained rate result

$$
\begin{gathered}
p_{i j}\left(\left\{\gamma_{k}\right\}, \varphi_{i}\right)=\left(\frac{\mu_{i}-\varphi_{i}}{\ln 2}-\frac{1}{\lambda_{i j}{ }^{2}\left(\left\{\gamma_{k}\right\}\right)}\right)^{+} i=1, \ldots, R \\
j=1, \ldots, m_{i} \\
r_{1 i}\left(\left\{\gamma_{k}\right\}, \varphi_{i}\right)=\sum_{j=1}^{m_{i}} \log _{2}\left(1+\lambda_{i j}{ }^{2}\left(\left\{\gamma_{k}\right\}\right) p_{i j}\left(\left\{\gamma_{k}\right\}, \varphi_{i}\right)\right)
\end{gathered}
$$

Proof. See Appendix 1.

Once we have the optimal solution for $\mathbf{T}_{i}$ 's and $\left\{r_{1 i}\right\}$ for a set of fixed $\left\{\gamma_{k}\right\}$ and $\left\{\varphi_{i}\right\}$, we have to optimize $\left\{\gamma_{k}\right\}$ and $\left\{\varphi_{i}\right\}$. The set $\left\{\varphi_{i}\right\}$ can be optimized applying the KKT conditions [19].

\section{Proposition 2}

Denoting by $\left\{\gamma_{k}\right\}$ a set of non-negative dual variables associated to the $k$ th per-BS power constraint, the optimal solution to $\left\{\varphi_{i}\right\}$ for a set of fixed $\left\{\gamma_{k}\right\}$ is given by 


$$
\varphi_{i}^{*}=\left\{\begin{array}{l}
\left.0 \quad \begin{array}{l}
\text { if } r_{1 i}\left(\left\{\gamma_{k}\right\}, 0\right) \leq \min \left(\alpha_{2} r_{2 i} / \alpha_{1}, S_{\max } m_{i}\right) \\
\mu_{i}-\ln 2 \cdot\left[2\left(\min \left(\alpha_{2} r_{2 i} / \alpha_{1}, S_{\max } m_{i}\right)-\sum_{j=1}^{m_{i}} \log _{2}\left(\lambda_{i j}^{2}\right)\right) / m_{i}\right.
\end{array}\right] \quad \text { otherwise } \quad i=1, \ldots, R
\end{array}\right.
$$

where the second result is obtained by substituting the expression of the transmission rate in (15) into the third constraint of problem $\left(P_{1}\right)$ and equaling the restriction. Notice that Equation (16) depends on the number of transmission modes per user $\left(m_{i}\right)$ and, in addition, we need to evaluate $r_{1 i}$ with Equation (15) which involves a water-filling algorithm. In this regard, an algorithm to find $\varphi_{i}^{*}$, which is based on hypothetic testing, is presented in Algorithm 1 (see Appendix 1 for details).

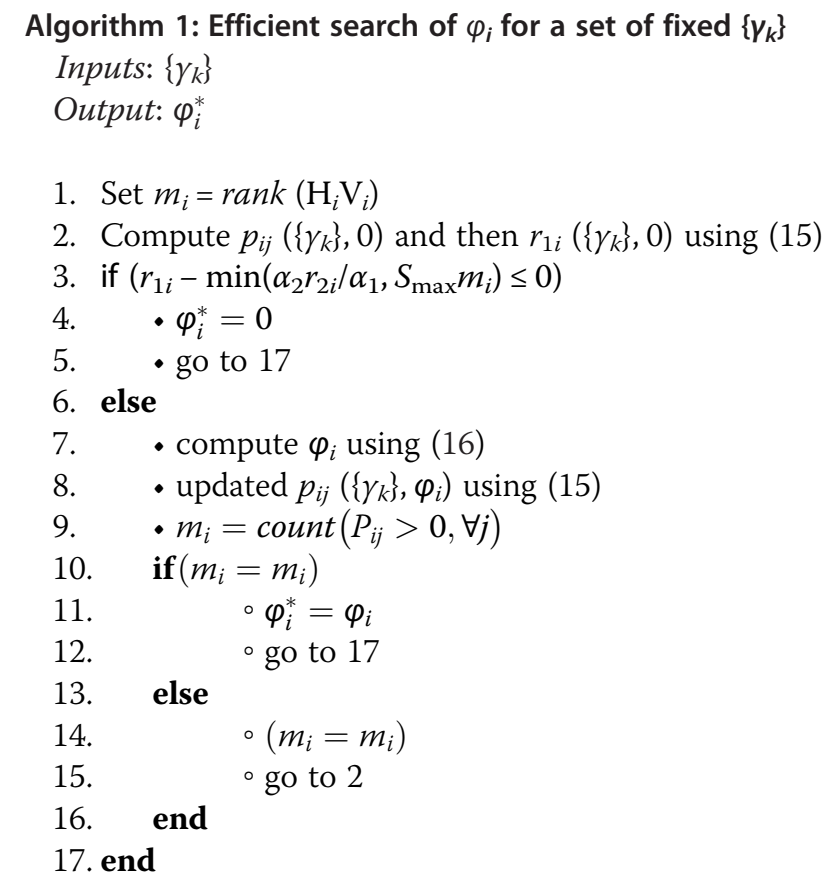

\section{Proof. See Appendix 1.}

Now, problem $\left(P_{1}\right)$ has to be optimized in terms of $\left\{\gamma_{k}\right\}$. In this regard, an effective algorithm is proposed in Algorithm 2 to compute $\left\{\gamma_{k}\right\}$ following the ellipsoid method [19]. For each set of $\left\{\gamma_{k}\right\}$ the optimal solution for $\mathbf{T}_{i}$ 's, $\left\{r_{1 i}\right\}$, and $\left\{\varphi_{i}\right\}$ are computed following (14), (15), and (16) (see details in Appendix 1).

\section{Algorithm 2: Efficient search of $\left\{\gamma_{k}\right\}$ and $\left\{\varphi_{i}\right\}$ in problem $P_{1}$}

1. Initialize: $\gamma_{k} \quad k=1, \ldots, B$

2. do

3. for $i=1, \ldots, R$

4. $\quad \circ \varphi_{i}^{*}=$ Algorithm $1\left(\left\{\gamma_{k}\right\}\right)$

$5 . \quad \circ$ Compute $\mathbf{T}_{i}$ using (14)

6. end
7. $\quad$ Compute the subgradient

$$
d_{k}=\operatorname{tr}\left(\sum_{i=1}^{R} \mathbf{B}_{k} \mathbf{V}_{i} \mathbf{T}_{i} \mathbf{V}_{i}^{H}\right)-P_{k}^{\max }
$$

8. - Update $\gamma_{k}{ }^{\prime}$ s accordingly based on the ellipsoid method [19]

9. until all $\gamma_{k}{ }^{\prime}$ s converge

10. Compute optimal $\mathbf{T}_{i}^{*}$ and $r_{1 i}$ given by (14) and (15).

Finally, from (14) we can directly obtain the optimal MIMO symbol precoding matrix:

$$
\mathbf{S}_{i}^{*}=\left(\mathbf{V}_{i}^{H} \mathbf{B}_{\gamma} \mathbf{V}_{i}\right)^{-1 / 2} \mathbf{W}_{i} \mathbf{P}_{i}^{1 / 2} \quad i=1, \ldots, R
$$

It is worth to remark that $\mathbf{S}_{i}$ " consists of non-orthogonal columns if $\mathbf{B}_{\gamma}$ is a non-identity diagonal matrix (i.e., the optimal $\left\{\gamma_{k}\right\}$ are not all equal). Moreover, it can be verified that for the sum-power constraint case, $\mathbf{S}_{i}{ }^{\prime \prime}$ in (17) contains orthogonal columns (i.e. orthogonal beamforming) since $\mathbf{V}_{i}^{H} \mathbf{V}_{i}=\mathbf{I}$, and hence its structure turns out to be independent of $\gamma$. For the same reason, when the optimal solution for $\mathbf{S}_{i}{ }^{*}(17)$ is used, the computational complexity is significantly higher than for the sum-power constraint case as singular value decomposition (SVD) must be computed for each algorithm's iteration (i.e., for each value of $\left\{\gamma_{k}\right\}$ ).

\subsection{WSR-based suboptimal resource allocation}

Let us assume a conventional precoding [15] for the MIMO symbol precoder $\mathbf{S}_{i}$ :

$$
\begin{aligned}
& \mathbf{S}_{i}=\hat{\mathbf{W}}_{i} \hat{\mathbf{P}}_{i}^{1 / 2} \quad i=1, \ldots, R \\
& \overline{\mathbf{H}}_{i}=\mathbf{N}_{i}^{-1 / 2} \mathbf{H}_{i} \mathbf{V}_{i} \\
& \hat{\mathbf{W}}_{i}=\operatorname{righteigenvec}\left(\overline{\mathbf{H}}_{i}\right), \\
& \hat{\mathbf{\Lambda}}_{i}=\operatorname{eigenval}\left(\overline{\mathbf{H}}_{i}\right)=\operatorname{diag}\left(\hat{\lambda}_{i 1} \cdots \hat{\lambda}_{i j} \cdots \hat{\lambda}_{i m_{i}}\right) \\
& \hat{\mathbf{P}}_{i}=\operatorname{diag}\left(\hat{p}_{i 1} \cdots \hat{p}_{i j} \cdots \hat{p}_{i m_{i}}\right)
\end{aligned}
$$

where $\hat{\mathbf{P}}_{i}$ is an $m_{i} \times m_{i}$ diagonal matrix describing the power allocated per symbol stream $\mathbf{b}_{i}$. It can be verified that the structure in (18) is optimal only when a single constraint (normally the sum-power) is imposed to the minimization problem $\left(P_{1}\right)$ in WSR-based optimal resource allocation. Even though, as we are interested in satisfying the per-BS power constraints, we can set the whole problem by including this structure for the MIMO symbol precoding matrices and then optimize the diagonal elements of $\hat{\mathbf{P}}_{i}$ to satisfy the per-BS power constraints and the MCS constraints. Following the 
precoding structure (18), the power transmitted by the $k$ th BS in (8) can be reformulated as

$$
\begin{aligned}
P_{k} & =\operatorname{tr}\left(\sum_{i=1}^{R} \widetilde{\mathbf{W}}_{i}^{k} \hat{\mathbf{p}}_{i} \widetilde{\mathbf{W}}_{i}^{k}\right) \\
& =\sum_{i=1}^{R} \sum_{j=1}^{m_{i}} \hat{p}_{i j} \widetilde{\mathbf{w}}_{i j}^{k H} \widetilde{\mathbf{w}}_{i j}^{k} \\
& =\sum_{i=1}^{R} \sum_{j=1}^{m_{i}} \hat{p}_{i j} \omega_{i j}^{k} \quad k=1, \ldots, B
\end{aligned}
$$

where $\tilde{\mathbf{W}}_{i}^{k}$ contains the $n_{\mathrm{B}}$ rows of $\mathbf{V}_{i} \hat{\mathbf{W}}_{i}$ used by the $k$ th $\mathrm{BS}$ in the transmission of message to the $i$ th RS and $\widetilde{\mathbf{w}}_{i j}^{k}$ is the $j$ th column of $\tilde{\mathbf{W}}_{i}^{k}$.

This way, the WSR maximization problem for the downlink transmission in relayed network-MIMO with BD-ZF precoding and per-BS power constraints can be formulated as follows [14]

$$
\begin{aligned}
& \left(P_{2}\right): \quad \text { minimize }-\sum_{i=1}^{R} \mu_{i} \hat{r}_{1 i} \\
& \text { s.t. }\left\{\begin{array}{l}
\sum_{i=1}^{R} \sum_{j=1}^{\left.m_{i}\right\},\left\{\hat{r}_{1 i}\right\}} \hat{r}_{i j} \omega_{i j}^{k}-P_{k}^{\max } \leq 0 \quad k=1, \ldots, B \\
\hat{r}_{1 i}-\sum_{j=1}^{m_{i}} \log _{2}\left(1+\hat{\lambda}_{i j}^{2} \hat{p}_{i j}\right) \leq 0 \quad i=1, \ldots, R \\
\hat{r}_{1 i}-\alpha_{2} r_{2 i} / \alpha_{1} \leq 0 \quad i=1, \ldots, R \\
\hat{p}_{i j}-\left(2^{S_{\max }}-1\right)\left(\hat{\lambda}_{i j}\right)^{-2} \leq 0 \quad i=1, \ldots, R \quad j=1, \ldots, m_{i} \\
-\hat{r}_{1 i} \leq 0 \quad i=1, \ldots, R \\
-\hat{p}_{i j} \leq 0 \quad i=1, \ldots, R \quad j=1, \ldots, m_{i}
\end{array}\right.
\end{aligned}
$$

where $\hat{r}_{1 i}$ is the transmission rate in the BS-RS link for the $i$ th UE and $\mu_{i}$ the priority given to it. The first constraint accounts for the per-BS power constraint, see (19), where $P_{k}^{\max }$ denotes the per-BS available power at the $k$ th $\mathrm{BS}$; the second constraint refers to the per-user transmission rate in the BS-RS link; the third constraint contains the per-user transmission rate in the RS-UE link (from Equation 12), and the fourth constraint is related to the per-user MCS constraint in the BS-RS link (which is here included directly to the power to be allocated). Finally, the fifth and sixth constraints account for the positiveness of the user's rate and allocated power.

Problem $\left(P_{2}\right)$ is convex and can be solved using standard convex optimization techniques, like interior point methods [19]. Nevertheless, in [14] we elaborate an efficient numerical algorithm based on the dual update methods $[19,20]$ which defines a polynomial complexity algorithm along with a reduction of the number of variables to be optimized.

\section{Proposition 3}

Denoting by $\left\{\phi_{k}\right\}$ and $\left\{\kappa_{i}\right\}$ a set of non-negative dual variables associated to the $k$ th per-BS power constraint and the $i$ th per-user transmission rate, respectively, the optimal solution to problem $\left(P_{2}\right)$ for a set of fixed $\left\{\phi_{k}\right\}$ and $\left\{\kappa_{i}\right\}$ is given by

$$
\begin{gathered}
\hat{p}_{i j}\left(\left\{\phi_{k}\right\}, \kappa_{i}\right)=\left[\frac{1}{\ln 2} \frac{\mu_{i}}{\sum_{k=1}^{B} \phi_{k} \omega_{i j}^{k}+\kappa_{i}}-\frac{1}{\hat{\lambda}_{i j}{ }^{2}}\right]_{0}^{\left(2^{\left.s_{\max }-1\right)\left(\hat{\lambda}_{i j}\right)^{-2}}\right.} \\
i=1, \ldots, R j=1, \ldots, m_{i} \\
\hat{r}_{1 i}\left(\left\{\phi_{k}\right\}, \kappa_{i}\right)=\sum_{j=1}^{m_{i}} \log _{2}\left(1+\hat{\lambda}_{i j}{ }^{2} \hat{p}_{i j}\left(\left\{\phi_{k}\right\}, \kappa_{i}\right)\right)
\end{gathered}
$$

Proof. See [14], where also an algorithm is proposed to effectively update $\left\{\phi_{k}\right\}$ and $\left\{\kappa_{i}\right\}$ in (21).

\section{Evaluations and results}

The evaluation of the proposed approach is done on a radio access network based on LTE-advanced specifications [3] at the $2 \mathrm{GHz}$ band and $20 \mathrm{MHz}$ bandwidth. We consider a hexagonal cellular deployment where $B=3$ BS and a total of $R=6$ RSs are deployed on the macrocell (see Figure 1). On each scenario, six UEs are dropped, each one attached to a different RS. Simulation parameters are detailed in Table 1.

Evaluations and results are taken in two different propagation scenarios: outdoor homogenous and outdoor/indoor hotspot. First, a deployment with simple propagation channel conditions is considered where users are located outdoor and homogeneously in the macrocell. Second, a hotspot scenario is deployed with indoor and outdoor users located forming areas of high traffic demand and propagation channel conditions are taken from the literature [21]. See Figure 2 for detailed deployment of users and RSs in the macrocell.

In relay-based systems, it is very important to optimize the duration of the relay-receive and the relay-transmit phases; but as we are assuming that they are fixed beforehand, it is crucial to make a good choice of the time devoted to each phase. In this regard, in [13] we observed that the optimum $\alpha_{1}$ in terms of spectral efficiency is a random variable that depends on the particular scenario and the target function to be maximized. For that reason, the duration of time slot $\alpha_{1}$ used in the simulations results is obtained from experimental results taking into account the LTE-A frame structure and the maximum delay for voice transmission [3]. The considered values are depicted in Table 2 for the different scenarios.

Two fundamental measures are adopted: cellular spectral efficiency $\left(S_{e}\right)$, as the sum rate of $R$ users divided by 3 (there are $R$ users and 3 cells deployed per macrocell) averaged over many deployments, and $95 \%$ outage rate $\left(r_{\text {out }}\right)$ (or cell-edge spectral efficiency), as the peak achievable rate of the 5-percentile worst users in the cell over many deployments. Both capture most of the benefits offered by coordination and cooperation of BS and relaybased transmission. 
Table 1 Overview of simulation parameters

\begin{tabular}{ll}
\hline Parameter & Value \\
\hline Central frequency & $2 \mathrm{GHz}$ \\
\hline $\begin{array}{l}\text { Channel bandwidth and duplex } \\
\text { method }\end{array}$ & $20 \mathrm{MHz}$ in TDD \\
\hline Macrocell order & $B=3$ \\
\hline Inter site distance (ISD) & $500 \mathrm{~m}$ \\
\hline Number of sectors & 3 \\
\hline Number of relays per sector & 2 (and hence $R=6)$ \\
\hline Sectors configurations & Convergent sectors \\
\hline Antenna height BS/RS/UE & $25 \mathrm{~m} / 10 \mathrm{~m} / 1.5 \mathrm{~m}$ \\
\hline Transmit power at BS/RS & $46 \mathrm{dBm} / 37 \mathrm{dBm}$ \\
\hline Noise spectral density & $-174 \mathrm{dBm} / \mathrm{Hz}$ \\
\hline Maximum number of beams & $n_{\mathrm{B}}=4$ \\
\hline Antenna elements at RS/UE & $n_{\mathrm{R}}=2 / n_{\mathrm{M}}=2$ (and thus $\left.m_{i}=2\right)$ \\
\hline BS/RS antenna gain & $14 \mathrm{dBi} / 5 \mathrm{dBi}$ \\
\hline Noise factor at BS and RS & 7 \\
\hline Noise figure at UE & $9 \mathrm{~dB}$ \\
\hline Total losses at BS/RS/UE & $6 \mathrm{~dB} / 2 \mathrm{~dB} / 2 \mathrm{~dB}$ \\
\hline
\end{tabular}

\subsection{Outdoor homogeneous scenario}

In a first deployment, all RSs are at the same distance $d_{B S-R S}$ to their associated BS, equal to $60 \%$ of the cell radius (experimentally found as the best position) and users are uniformly placed beyond $60 \%$ of the cell radius (so as to include only those users benefiting from the presence of relays). See Figure 2-left for detailed deployment of the RS and users in the macrocell.

The propagation channel between each BS and RS, and between each RS's and UE's is characterized by a path loss component, a shadow fading and a Rayleigh fading. The path loss component is modeled as $\beta\left(d / d_{\mathrm{o}}\right)^{-\rho}$, where $d$ is the distance in kilometer between transmitter and receiver, $d_{o}$ is the reference distance (equal to $1 \mathrm{~km}$ ), $\beta$ is the propagation constant (equal to $\left(4 \pi f_{c} /\left(3 \times 10^{8}\right)\right)^{2}$ for BS-RS links and equal to $2 \pi f_{c} /\left(3 \times 10^{8}\right)$ for RS-UE links $)$ and $\rho$ denotes the path loss propagation exponent which is also different for each link; assuming a LOS situation of RS with respect to BS: $\rho_{B S-R S}=2$ (which then defines the free space propagation model), and $\rho_{R S-U E}$ is an input variable for our simulations. The shadowing effect is assumed Gaussian in logarithmic scale with a standard deviation of $4 \mathrm{~dB}$ for BS-RS link and $8 \mathrm{~dB}$ for RS-UE link. Therefore, the channel from the $l$ th transmit antenna of the $k$ th transmitter and the $m$ th receive antenna of the $i$ th receiver is given by

$$
h_{i, k}^{m, l}=\delta_{i, k}^{m, l} \sqrt{\chi_{i, k} \beta\left(d_{i, k} / d_{o}\right)^{-\rho}}
$$

where $\chi_{i, k}$ is the shadowing between the $k$ th transmitter and the $i$ th receiver, and $\delta_{i, k}{ }^{m, l} \in C N(0,1)$ represents the Rayleigh fading.

In this section, the following transmission strategies are evaluated:

- Relayed network-MIMO optimal: relay-based transmission with cooperative BSs using networkMIMO and the optimal precoding strategy derived in WSR-based optimal resource allocation.

- Relayed network-MIMO suboptimal: relay-based transmission with cooperative BSs using networkMIMO and the suboptimal precoding strategy described in WSR-based suboptimal resource allocation.
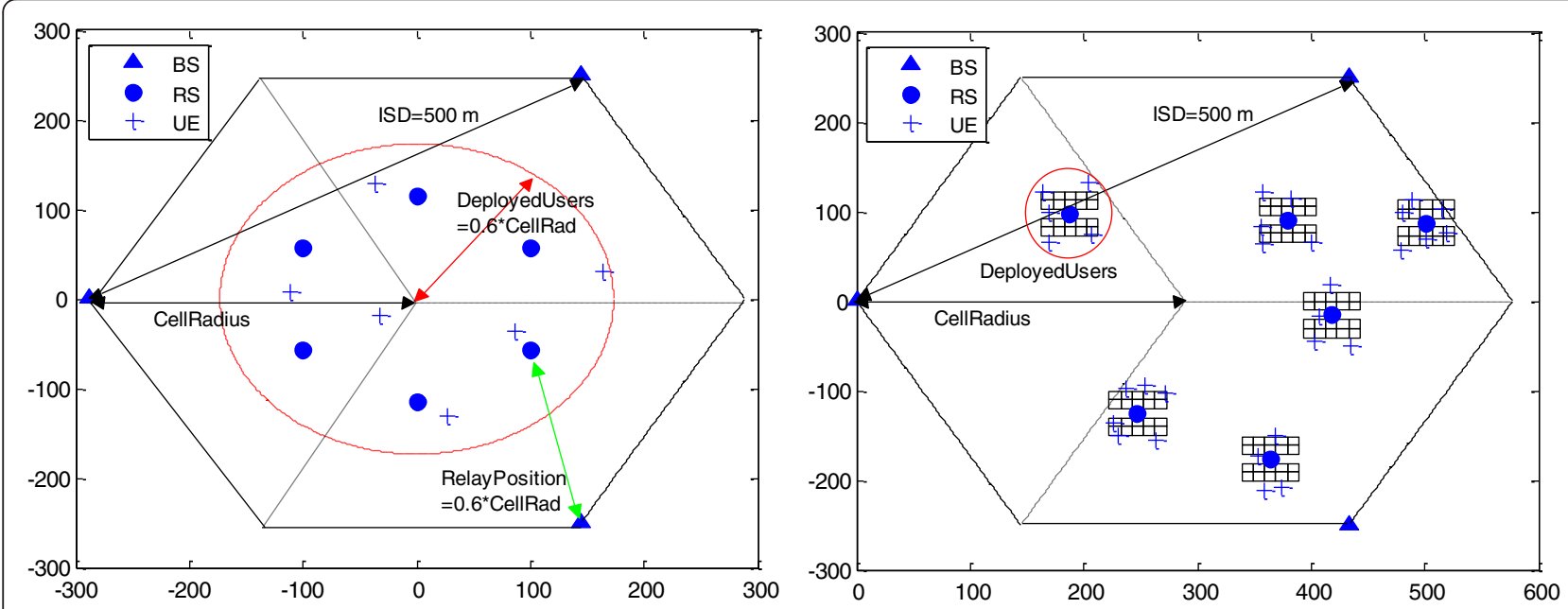

Figure 2 Macrocell and users' deployment in the homogeneous scenario (left) and in the hotspot scenario (right). 
Table 2 Duration of the relay-receive phase for simulation results

\begin{tabular}{ll}
\hline Homogeneous scenario & $\mathbf{a}_{\mathbf{1}}$ (BS-RS link) \\
\hline Relayed network-MIMO & \\
\hline Relayed BDZF-TDMA & $1 / 8$ \\
\hline Relayed BF-TDMA & $2 / 8$ \\
\hline Hotspot scenario & $3 / 8$ \\
\hline Relayed network-MIMO with MCS constraints & $4 / 8$ \\
\hline Relayed BDZF-TDMA with MCS constraints & $6 / 8$ \\
\hline
\end{tabular}

- Relayed BDZF-TDMA: relay-based transmission with non-cooperative BSs, where one time slot is allocated per BS transmission and each BS serves simultaneously its associated relays following a multiuser MIMO BD-ZF strategy [17].

- Relayed BF-TDMA: relay-based transmission with non-cooperative BSs, where each BS serves its associated relays under round-robin TDMA.

These transmission strategies are evaluated for the SR over 1,000 random user deployments when no MCS constraints are taken into account.

Figure 3 displays $S_{e}$ versus the path loss propagation exponent of the RS-UE link $\left(\rho_{R S-U E}\right)$. On the one side, significant gains are observed in terms of $S_{e}$ when using network-MIMO strategies in the BS-RS link as compared to the utilization of non-cooperative BS-based strategies (BDZF-TDMA or BF-TDMA). On the other side, the performance gain achieved by the suboptimal MIMO symbol precoding is near to the gain obtained with the optimal precoding (moreover, the difference is depreciable when the system is limited by transmission in the RS-UE link), while the computational complexity is significantly increased when using the optimal precoding strategy. Regarding the performance in terms of propagation exponent of the second hop, $\rho_{R S-U E}$, the performance is nearly constant for low values of $\rho_{R S-U E}$ because of the high level of received interference from non-serving RS. As the attenuation in the RS-UE link increases (i.e., higher values of $\left.\rho_{R S-U E}\right)$, the system performance is severely degraded.

Figure 4 displays $S_{e}$ versus the time devoted for BS-RS links $\left(\alpha_{1}\right)$ when $\rho_{R S-U E}=3$. Relay-based transmission strategies are compared to the case where both precoders and durations for the relay-transmit and relay-receive phases are optimized on each deployment following a networkMIMO suboptimal precoding strategy and considering that interference is stationary during the transmission intervals, as it was done in [13]. However, let us remark that optimizing the duration of the phases on each realization is not possible in practice, since the interference between

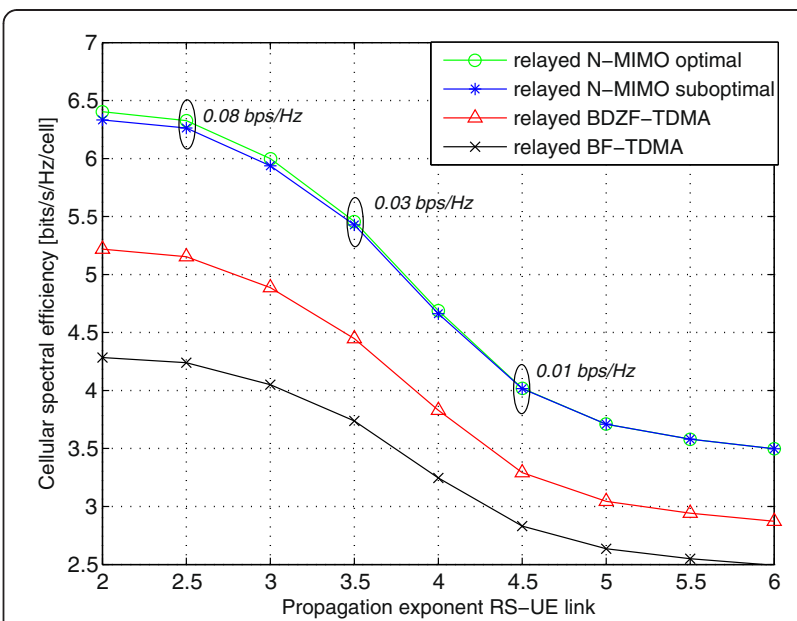

Figure 3 Cellular spectral efficiency $\left(S_{e}\right)$ versus path loss propagation exponent in RS-UE link $\left(\rho_{R S-U E}\right)$ for different transmission schemes in BS-RS link.

macrocells would be time-varying. In Figure 4, it can be observed that, for each transmission strategy, there exists an optimum value for the transmit duration phases so as to maximize the cellular spectral efficiency in average. These values of $\alpha_{1}$ are the ones used for the system evaluations, as it is explicitly detailed in Table 2. Furthermore, when comparing the 'relayed network-MIMO optimal and suboptimal' with the upper bound given by the joint optimization of the precoders and phase durations on each realization, it can be observed that the difference is not significant when properly selecting $\alpha_{1}$.

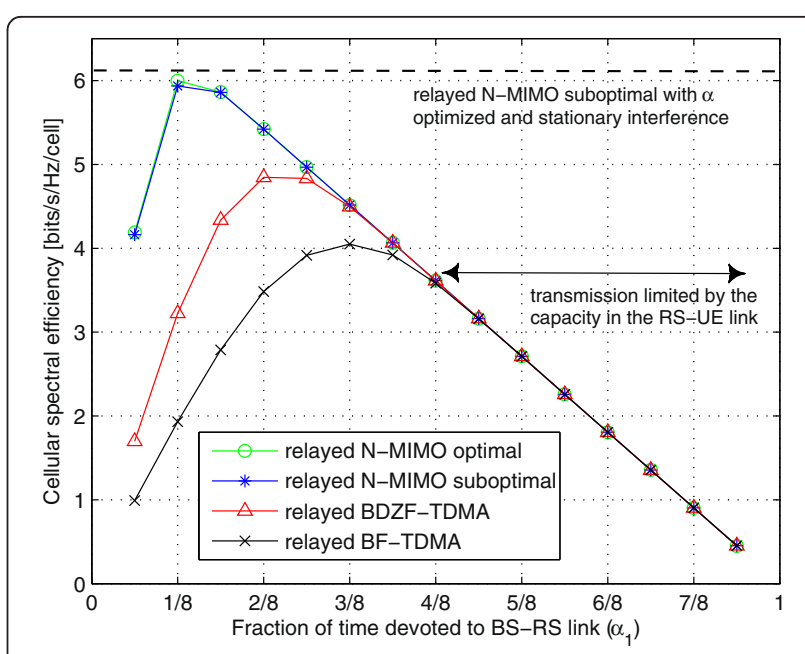

Figure 4 Cellular spectral efficiency $\left(S_{e}\right)$ versus fraction of time devoted to BS-RS links $\left(a_{1}\right)$ for different transmission schemes in BS-RS link when $\rho_{R S-U E}=3$. 
Finally, in Figure 5, the capacity of individual BS-RS link is evaluated when the path loss propagation exponent of the BS-RS link $\left(\rho_{B S-R S}\right)$ increases, without taking into account the constraints imposed by the RS-UE link capacity and the maximum MCS. We can observe a gain in the capacity of 300 and $600 \%$ due to the cooperation of BSs, when comparing network-MIMO schemes to BD-ZF TDMA and BF-TDMA, respectively. In addition, comparing the optimal and suboptimal precoding structures, we can observe that the gain obtained by the suboptimal scheme is very close to that achieved with the optimal scheme. Results show that there is a constant gain irrespective of channel propagation conditions. This confirms the fact that when a relay-based transmission is limited by the capacity in the RS-UE link, the optimal precoding does not obtain a significant enhanced result compared to the suboptimal case.

To conclude, in all studies it is observed that the suboptimal precoding strategy for a relayed network-MIMO system nearly achieves the optimum solution. Thanks to the evaluation of the BS-RS capacity in Figure 5, it is verified that the similar performance between both solutions is due to the first hop precoding strategy itself and not due to the inclusion of RS-UE link constraints or maximum MCS constraints. However, we do not have a mathematical intuition to explain the similar performance between both precoding strategies, since it is very difficult to relate the eigenvalues of $\mathbf{N}_{i}^{-1 / 2} \mathbf{H}_{i} \mathbf{V}_{i}$ in (18) with the eigenvalues of $\mathbf{N}_{i}^{-1 / 2} \mathbf{H}_{i} \mathbf{V}_{i}\left(\mathbf{V}_{i}^{H} \mathbf{B}_{\gamma} \mathbf{V}_{i}\right)^{-1 / 2}$ in (14), whose values depend on the Lagrange multipliers associated to the power constraints $\left\{\gamma_{k}\right\}$ for which there is not an analytical expression.

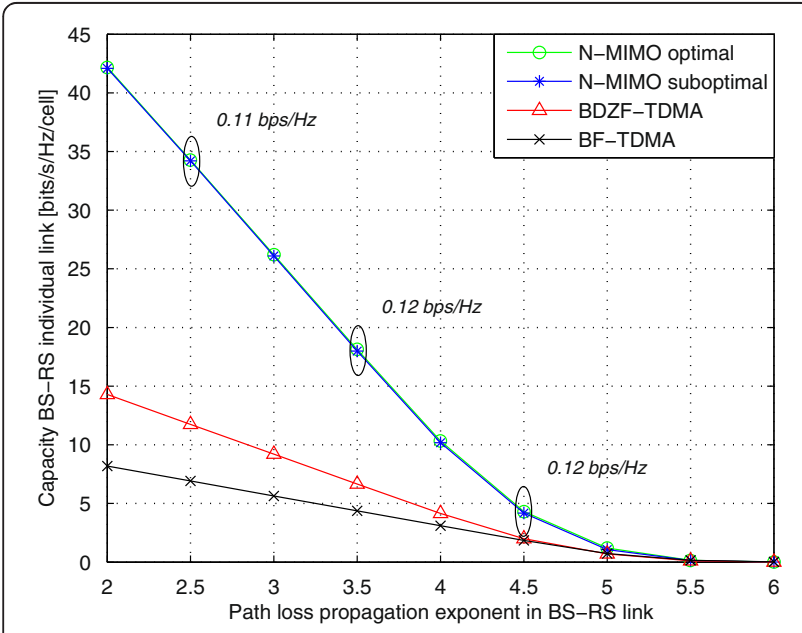

Figure 5 Capacity BS-RS link versus path loss propagation exponent in BS-RS link $\left(\rho_{B S-R S}\right)$ for different transmission schemes.

\subsection{Outdoor/indoor hotspot scenario}

In a second deployment, a hotspot scenario is considered to evaluate a realistic and more complex deployment, where users are located in indoor/outdoor areas of high traffic demand. Each hotspot is modeled as two buildings (with 10 apartments per floor and several floors) and the streets between them, following the specifications of the dual stripe model for corporate urban deployments [22] (see minor details in Table 3). One relay is placed on each hotspot. See Figure 2-right for detailed deployment of the RS and users in the macrocell. In addition, two tiers of surrounding macrocells are considered as interference for each macrocell, and the worst-case scenario is considered: interfering BSs transmit at their maximum allocated power.

Channel models adopted are obtained from [21]. We assume LOS conditions for all BS-RS links, distancedependent LOS/NLOS conditions for transmissions from BS and RS to outdoor UE and NLOS conditions with wall penetration losses for transmissions from BS and RS to indoor UE. See details of the models used in Table 3.

In this section, the following transmission strategies are evaluated:

- Relayed network-MIMO optimal: relay-based transmission with cooperative BSs using networkMIMO and the optimal precoding strategy derived in WSR-based optimal resource allocation.

- Relayed network-MIMO suboptimal: relay-based transmission with cooperative BSs using networkMIMO and the suboptimal precoding strategy described in WSR-based suboptimal resource allocation.

- Relayed BDZF-TDMA: relay-based transmission with non-cooperative BSs, where one time slot is allocated per BS transmission and each BS serves simultaneously its associated relays following a multiuser MIMO BD-ZF strategy [17].

- Direct network-MIMO optimal: non-relayed transmission with cooperative BSs using networkMIMO and the optimal precoding strategy [12].

- Direct network-MIMO suboptimal: non-relayed transmission with cooperative BSs using networkMIMO and the suboptimal precoding strategy.

These transmission strategies are evaluated for the SR and for the WSR over 1,000 random user deployments when taking into account the MCS constraints (from LTE-A specifications [3]: $S_{\max }=4.8 \mathrm{bps} / \mathrm{Hz}$ ). When using WSR, the weights are inversely proportional to the rates in the second hop (i.e., $\mu_{i}=1 / r_{2 i}$ ), in order to avoid unfair service to deprived users. 
Table 3 Overview of deployment parameters and channel models for a hotspot scenario

\begin{tabular}{|c|c|c|}
\hline \multicolumn{2}{|l|}{ Parameter } & Value \\
\hline \multicolumn{2}{|c|}{ Number of areas per cell } & 2 \\
\hline \multicolumn{2}{|l|}{ Area composition } & $\begin{array}{l}\text { Two buildings with several floors, } \\
\text { three streets }\end{array}$ \\
\hline \multicolumn{2}{|c|}{ Number of offices on each floor } & 10 \\
\hline \multicolumn{2}{|l|}{ Office size } & $10 \mathrm{~m} \times 10 \mathrm{~m}$ \\
\hline \multicolumn{2}{|l|}{ Office height } & $3 \mathrm{~m}$ \\
\hline \multicolumn{2}{|l|}{ Street weight } & $10 \mathrm{~m}$ \\
\hline \multicolumn{2}{|c|}{$\begin{array}{l}\text { Probability of the user to be } \\
\text { outdoor }\end{array}$} & 0.8 \\
\hline \multirow{5}{*}{ Path loss models } & BS to RS & B5a LOS and C1 NLOS Winner II \\
\hline & $\begin{array}{l}\text { BS to UE } \\
\text { outdoor }\end{array}$ & C2 (UMa) Winner II \\
\hline & $\begin{array}{l}\text { BS to UE } \\
\text { indoor }\end{array}$ & Dense urban indoor \\
\hline & $\begin{array}{l}\text { RS to UE } \\
\text { outdoor }\end{array}$ & $\begin{array}{l}\text { ITU UMi with 10-m transmit } \\
\text { antenna height }\end{array}$ \\
\hline & $\begin{array}{l}\text { RS to UE } \\
\text { indoor }\end{array}$ & C4 Winner II \\
\hline \multicolumn{2}{|l|}{ MIMO channel } & $\begin{array}{l}\text { LOS and NLOS angular } \\
\text { dispersion used }\end{array}$ \\
\hline \multicolumn{2}{|c|}{ Shadowing \& fast fading } & $\begin{array}{l}\text { Distance-dependent shadowing, } \\
\text { from Winner }\end{array}$ \\
\hline \multirow{3}{*}{$\begin{array}{l}\text { Shadowing standard } \\
\text { deviation }\end{array}$} & BS to RS & B5a LOS and C1 NLOS Winner II \\
\hline & BS to UE & ITU UMa/C2 Winner II \\
\hline & RS to UE & ITU UMi/C4 Winner II \\
\hline \multicolumn{2}{|c|}{ Antenna array/elements at BS } & ULA/4-12 \\
\hline \multicolumn{2}{|c|}{ Penetration loss due to buildings } & $10 \mathrm{~dB}$ \\
\hline
\end{tabular}

Figure 6 displays $r_{\text {out }}$ versus $S_{e}$ for ISD of 500 and $1500 \mathrm{~m}$, while Figure depicts the cumulative density function (CDF) of the user's transmission rate for ISD equal to $500 \mathrm{~m}$.

Figures 6 and 7 elucidate that deploying RS significantly improves the system coverage in a corporate hotspot scenario (for both indoor and outdoor users), as the outage rate is higher for both relay-assisted transmissions (based on network-MIMO and BDZF-TDMA) compared to the results achieved in a transmission without relays (direct network-MIMO). It is due to the fact that RS are nearer the UE and they are able to efficiently combat shadowing and path loss effects. Moreover, in [14] it is shown that the enhanced cell-edge spectral efficiency and coverage thanks to RS's deployment is achieved with a lower transmitted power as compared to direct transmissions. Notice that the $95 \%$ outage rate is 0 in the direct transmission evaluated with network-MIMO and SR criteria, because there are a high number of users with bad propagation channel conditions. We observed the same results even assuming the WSR criterion.
When comparing the relay-assisted transmissions in Figure 6 ('relayed network-MIMO' and 'relayed BDZFTDMA'), network-MIMO achieves a nearly doubled cellular spectral efficiency. BDZF-TDMA suffers some loses in spectral efficiency due to the time division associated to coordination. In addition, it achieves an improved cell-edge rate because the duration of the relay-receive and relay-transmit phases is optimized beforehand (see Table 2) on each transmission scheme to enhance the system spectral efficiency (and they differ from networkMIMO to BDZF-TDMA).

If we compare the relay-assisted transmissions with cooperation of BSs for BS-RS link ('relayed networkMIMO' in legend) when using the optimal and the suboptimal precoding structure, it can be observed that when using the optimal structure results in terms of spectral efficiency and outage rate are slightly enhanced. It has been observed that these results are achieved with a significantly increased computational complexity.

When comparing results for relayed network-MIMO with different object functions ('SR' or 'WSR' in Figure 6), it can be observed that WSR criterion performs better in terms of outage rate while it suffers some losses in terms of spectral efficiency, as it is more concerned about users with bad propagation channel conditions in the RS-UE link.

In Figure 7, we can observe the effect of the MCS constraints over the half-duplex relay-assisted transmissions, which are applied to each link of the RS-based transmission. Hence, the maximum per-user transmission rate that can be achieved by a direct transmission

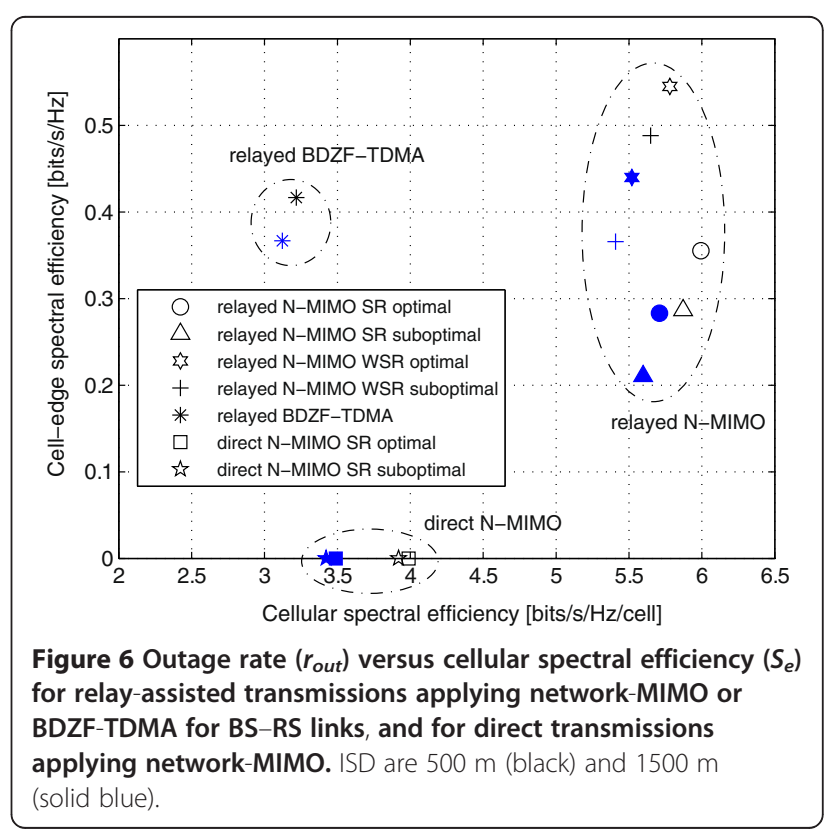




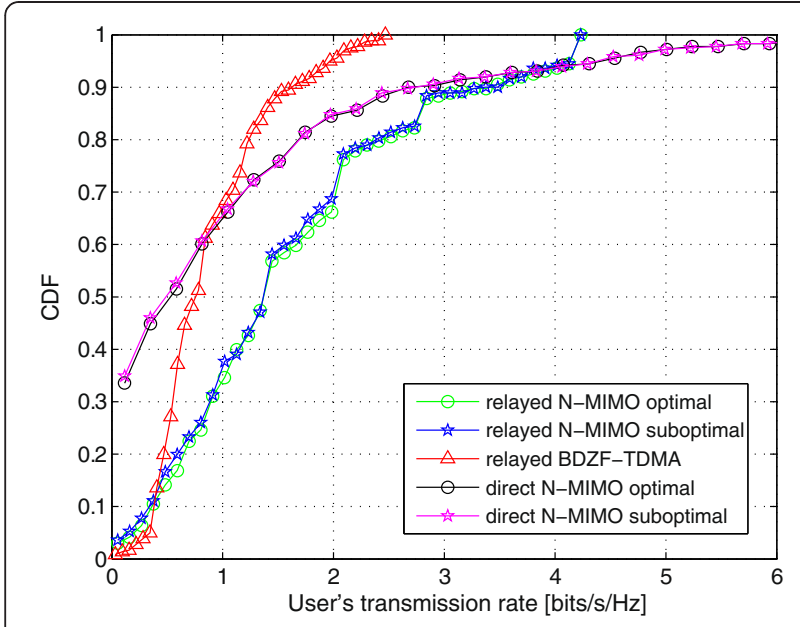

Figure $7 \mathrm{CDF}$ of users' transmission rates for relay-assisted transmissions applying network-MIMO or BDZF-TDMA for BS-RS links, and for direct transmissions applying network-MIMO. ISD is $500 \mathrm{~m}$.

is nearly doubled than for a relay-based transmission (even in the scenario deployed, it is achieved by less than $95 \%$ of the users).

To conclude, in a hotspot or corporate scenario, deploying relays using network-MIMO in the BS-RS link represents a competitive solution for enhanced services, since it allows improving cellular spectral efficiency and outage rate compared to a non-relayed transmission with cooperating BSs or a relay-based transmission with no cooperating BSs.

\section{Conclusions}

This article derives the optimal MIMO symbol precoding matrix and power allocation for QoS-constrained relay-assisted cellular systems, where cooperation between BS is appointed and the duration of the relaying phases is fixed beforehand. Results in terms of spectral efficiency and outage rate with the optimal MIMO symbol precoding matrix have been obtained and shown not to be significantly better than those obtained with the suboptimal precoding, which requires a lowcomplexity algorithm and lower computational time. Moreover, the suitability of applying network-MIMO in the BS-RS transmission has been proved in a corporate hotspot scenario. Enhanced results in cellular spectral efficiency and outage rate are obtained compared to non-relayed transmission or relayed transmission with non-cooperative BSs. Further work is oriented to study modulation-constrained resource allocation for the uplink, user's grouping and scheduling strategies, and coordination of transmissions between cell clusters.

\section{Appendix 1}

The Lagrangian function of problem $\left(P_{1}\right)$ in $(13)$ can be written as

$$
\begin{aligned}
& L\left(\left\{r_{1 i}\right\},\left\{\mathbf{T}_{i}\right\},\left\{\gamma_{k}\right\},\left\{\psi_{i}, \varphi_{i}, \eta_{i}\right\}\right) \\
& =\sum_{i=1}^{R} \hat{L}_{i}\left(r_{1 i}, \mathbf{T}_{i},\left\{\gamma_{k}\right\}, \psi_{i}, \varphi_{i}, \eta_{i}\right) \\
& \quad-\sum_{k=1}^{B} \gamma_{k} P_{k}^{\max } \\
& \quad-\sum_{i=1}^{R} \varphi_{i} \min \left(\alpha_{2} r_{2 i} / \alpha_{1}, S_{\max } m_{i}\right)
\end{aligned}
$$

where $\hat{L}_{i}$ contains the terms which depend on $\left\{r_{1 i}\right\}$ and $\mathbf{T}_{i}^{\prime} \mathbf{s}$

$$
\begin{aligned}
\hat{L}_{i}\left(r_{1 i}, \mathbf{T}_{i},\left\{\gamma_{k}\right\}, \psi_{i}, \varphi_{i}, \eta_{i}\right) \\
=\left(-\mu_{i}+\psi_{i}+\varphi_{i}-\eta_{i}\right) r_{1 i}+\operatorname{tr}\left(\mathbf{B}_{\gamma} \mathbf{V}_{i} \mathbf{T}_{i} \mathbf{V}_{i}^{H}\right) \\
\quad-\psi_{i} \log _{2}\left|\mathbf{I}+\mathbf{N}_{i}^{-1 / 2} \mathbf{H}_{i} \mathbf{V}_{i} \mathbf{T}_{i} \mathbf{V}_{i}^{H} \mathbf{H}_{i}^{H} \mathbf{N}_{i}^{-H / 2}\right|
\end{aligned}
$$

where $\mathbf{B}_{\gamma}=\sum_{k=1}^{B} \gamma_{k} \mathbf{B}_{k} ; \gamma_{k}, \psi_{i}$, and $\varphi_{i}$ denote the Lagrange multipliers or dual variables associated to the per-BS power constraint, the per-user transmission rate in the BS-RS link, and the per-user transmission rate, respectively. Finally, $\eta_{i}$ is the Lagrange multiplier needed for having positive values of transmission rates.

If we formulate the Lagrange dual function of problem $\left(P_{1}\right)$

$$
g\left(\left\{\gamma_{k}\right\},\left\{\psi_{i}, \varphi_{i}, \eta_{i}\right\}\right)=\underset{\left\{r_{1 i}\right\},\left\{\mathbf{T}_{i}\right\}}{\operatorname{minimize}} L\left(\left\{r_{1 i}\right\},\left\{\mathbf{T}_{i}\right\},\left\{\gamma_{k}\right\},\left\{\psi_{i}, \varphi_{i}, \eta_{i}\right\}\right)
$$

Formally, the dual function should be defined as the infimum of the Lagrangian function, but in our case the infimum coincides with the minimum since the infimum is contained in the convex set [19]. This way, we can define the dual problem of $\left(P_{1}\right)$ as

$$
\left(P_{\mathrm{DP}}\right): \quad \operatorname{maximize}_{\psi_{i}, \gamma_{k}, \varphi_{i}, \eta_{i} \geq 0} g\left(\left\{\gamma_{k}\right\},\left\{\psi_{i}, \varphi_{i}, \eta_{i}\right\}\right)
$$

Since $\left(P_{\mathrm{DP}}\right)$ is convex and satisfies the Slater's condition [19], the duality gap between the optimal objective value of $\left(P_{\mathrm{DP}}\right)$ and $\left(P_{1}\right)$ is zero. Then, $\left(P_{1}\right)$ can be solved equivalently by solving $\left(P_{\mathrm{DP}}\right)$. Now, we focus on solving 
$\left\{r_{1 i}\right\}$ and $\mathbf{T}_{i}$ 's for a set of fixed $\left\{\gamma_{k}\right\},\left\{\psi_{i}\right\},\left\{\varphi_{i}\right\}$, and $\left\{\eta_{i}\right\}$, as it is expressed in Equation (25). Notice that Equation (25) can be separated into $R$ independent minimization subproblems (on for each UE, see (23)) and hence we focus on

$$
\underset{r_{1 i}, \mathbf{T}_{i}}{\operatorname{minimize}} \hat{L}_{i}\left(r_{1 i}, \mathbf{T}_{i},\left\{\gamma_{k}\right\}, \psi_{i}, \varphi_{i}, \eta_{i}\right) \quad i=1, \ldots, R
$$

If we define

$$
\widetilde{\mathbf{T}}_{i}=\left(\mathbf{V}_{i}^{H} \mathbf{B}_{\gamma} \mathbf{V}_{i}\right)^{1 / 2} \mathbf{T}_{i}\left(\mathbf{V}_{i}^{H} \mathbf{B}_{\gamma} \mathbf{V}_{i}\right)^{1 / 2}
$$

the minimization problem in (27) can be reformulated as

$$
\begin{aligned}
\underset{r_{1 i}, \widetilde{\mathbf{T}}_{i}}{\operatorname{minimize}} & \left(-\mu_{i}+\psi_{i}+\varphi_{i}-\eta_{i}\right) r_{1 i}+\operatorname{tr}\left(\widetilde{T}_{i}\right) \\
-\psi_{i} \log _{2} \mid & \mathbf{I}+\mathbf{N}_{i}^{-1 / 2} \mathbf{H}_{i} \mathbf{V}_{i}\left(\mathbf{V}_{i}^{H} \mathbf{B}_{\gamma} \mathbf{V}_{i}\right)^{-1 / 2} \\
& \times \widetilde{\mathbf{T}}_{i}\left(\mathbf{V}_{i}^{H} \mathbf{B}_{\gamma} \mathbf{V}_{i}\right)^{-1 / 2} \mathbf{V}_{i}^{H} \mathbf{H}_{i}^{H} \mathbf{N}_{i}^{-H / 2} \mid
\end{aligned}
$$

On the one hand, the condition to minimize (29) as a function of $r_{1 i}$ is

$$
\partial L / \partial r_{1 i}=0 \rightarrow-\mu_{i}+\psi_{i}+\varphi_{i}-\eta_{i}=0
$$

On the other hand, the solution to minimize (29) as a function of $\hat{\mathbf{T}}_{i}$ can be found applying Hadamard's inequality [23]. Since the logarithm is an increasing function, it is the same maximizing the logarithm than the values inside it. Hadamard's inequality demonstrates that the optimal solution for those problems is such that diagonalizes the values inside the determinant of (27). Therefore, if we consider the following (reduced) SVD

$$
\mathbf{N}_{i}^{-1 / 2} \mathbf{H}_{i} \mathbf{V}_{i}\left(\mathbf{V}_{i}^{H} \mathbf{B}_{\gamma} \mathbf{V}_{i}\right)^{-1 / 2}=\mathbf{U}_{i} \boldsymbol{\Lambda}_{i} \mathbf{W}_{i}^{H}
$$

where $\boldsymbol{\Lambda}_{i}=\operatorname{diag}\left(\lambda_{i 1}, \ldots, \lambda_{i m_{i}}\right)$ is an $m_{i} \times m_{i}$ diagonal matrix containing the positive eigenvalues and $\mathbf{U}_{i}$ and $\mathbf{W}_{i}$ are unitary matrix containing the $m_{i}$ left singular vectors and the $m_{i}$ right singular vectors, respectively. Then, the optimal solution for (27) for a set of fixed $\left\{\gamma_{k}\right\}$, $\left\{\psi_{i}\right\},\left\{\varphi_{i}\right\}$, and $\left\{\eta_{i}\right\}$ is

$$
\widetilde{\mathbf{T}}_{i}=\mathbf{W}_{i} \mathbf{P}_{i} \mathbf{W}_{i}^{H} \quad i=1, \ldots, R
$$

where $\mathbf{P}_{i}=\operatorname{diag}\left(p_{i 1}, \ldots, p_{i m_{i}}\right)$ is a diagonal matrix, which diagonal values can be obtained from the standard waterfilling algorithm [23], when also considering $\psi_{i}=\mu_{i}-\varphi_{i}$ from (30)

$$
\begin{aligned}
p_{i j}\left(\left\{\gamma_{k}\right\}, \varphi_{i}\right) & =\left(\frac{\mu_{i}-\varphi_{i}}{\ln 2}-\frac{1}{\lambda_{i j}{ }^{2}\left(\left\{\gamma_{k}\right\}\right)}\right)^{+} \\
i & =1, \ldots, R \quad j=1, \ldots, m_{i}
\end{aligned}
$$

It can be verified that (32) satisfies the channel diagonalization property. Therefore, denoted $\left\{\gamma_{k}\right\}$ and $\left\{\varphi_{i}\right\}$ a set of non-negative dual variables associated to the $k$ th per-BS power constraint and the $i$ th per-user transmission rate, respectively, the optimal solution to (27) for fixed $\left\{\gamma_{k}\right\}$ and $\left\{\varphi_{i}\right\}$ is given by

$$
\begin{aligned}
& \mathbf{T}_{i}^{*}=\left(\mathbf{V}_{i}^{H} \mathbf{B}_{\gamma} \mathbf{V}_{i}\right)^{-1 / 2} \mathbf{W}_{i} \mathbf{P}_{i} \mathbf{W}_{i}{ }^{H}\left(\mathbf{V}_{i}^{H} \mathbf{B}_{\gamma} \mathbf{V}_{i}\right)^{-1 / 2} \\
& r_{1 i}\left(\left\{\gamma_{k}\right\}, \varphi_{i}\right) \\
& =\sum_{j=1}^{m_{i}} \log _{2}\left(1+\lambda_{i j}^{2}\left(\left\{\gamma_{k}\right\}\right) p_{i j}\left(\left\{\gamma_{k}\right\}, \varphi_{i}\right)\right) \quad i=1, \ldots, R
\end{aligned}
$$

Once we have the optimal solution for $\mathbf{T}_{i}$ 's and $\left\{r_{1 i}\right\}$ for a set of fixed $\left\{\gamma_{k}\right\}$ and $\left\{\varphi_{i}\right\}$, we have to optimize $\left\{\gamma_{k}\right\}$ and $\left\{\varphi_{i}\right\}$ to obtain the optimal solution to $\left(P_{\mathrm{DP}}\right)$.

On the one hand, we can apply the Karush-KuhnTucker conditions [19] to obtain the optimal $\left\{\varphi_{i}\right\}$ for each set of fixed $\left\{\gamma_{k}\right\}$. Specifically, the Slater's condition [19] over the per-user transmission rate specifies

$$
\varphi_{i}\left(r_{1 i}-\min \left(\alpha_{2} r_{2 i} / \alpha_{1}, S_{\max } m_{i}\right)\right)=0 \quad i=1, \ldots, R
$$

So, if the resource allocation for the $i$ th user is nonlimited by the per-user transmission rate (i.e., $r_{1 i}<\mathrm{min}$ $\left(\alpha_{2} r_{2 i} / \alpha 1, S_{\max } m_{i}\right)$ then $\varphi_{i}=0$. Otherwise, if the resource allocation for the $i$ th user is limited by the per-user transmission rate, we need to impose $r_{1 i}=\min \left(\alpha_{2} r_{2 i} / \alpha 1\right.$, $\left.S_{\max } m_{i}\right)$ and $\varphi_{i}$ can be obtained from the imposition when substituting (33) into $r_{1 i}$ following (34). Then, the optimal value for $\left\{\varphi_{i}\right\}$ for a set of fixed $\left\{\gamma_{k}\right\}$ can be expressed as 


$$
\varphi_{i}^{*}=\left\{\begin{array}{l}
0 \quad \text { if } r_{1 i}\left(\left\{\gamma_{k}\right\}, 0\right) \leq \min \left(\alpha_{2} r_{2 i} / \alpha_{1}, S_{\max } m_{i}\right) \\
\mu_{i}-\ln 2 \cdot\left[2\left(\min \left(\alpha_{2} r_{2 i} / \alpha_{1}, S_{\max } m_{i}\right)-\sum_{j=1}^{m_{i}} \log _{2}\left(\lambda_{i j}^{2}\right)\right) / m_{i}\right] \text { otherwise } \quad i=1, \ldots, R
\end{array}\right.
$$

Notice that the $\varphi_{i}^{*}$ depends on the number of transmission modes per user $\left(m_{i}\right)$ and, in addition, we need to evaluate $r_{1 i}$ with Equation (34) which involves a water-filling algorithm for the power allocation. For that reason, the optimal value for $\varphi_{i}$ cannot directly be found. In this regard, an algorithm to find $\varphi_{i}^{*}$, which is based on hypothetic testing is presented in Algorithm 1. The underlying idea is to form a hypothesis of the active and inactive transmission modes and check whether a consistent solution can be found or not. In the Algorithm 1, initially it is assumed that all transmissions modes are active (and hence $\left.m_{i}=\operatorname{rank}\left(\mathbf{H}_{i} \mathbf{V}_{i}\right) i=1, \ldots, R\right)$; then, $\varphi_{i}^{\prime \prime}$ is computed following (36) and the power allocation is calculated with (33); in the case that the resulting active transmission modes due to the power allocation are lower than those initially supposed, we have to reduce the number of active transmission modes and make a new hypothesis; until consistence.

On the other hand, problem $\left(P_{\mathrm{DP}}\right)$ has to be optimized in terms of $\left\{\gamma_{k}\right\}$. The optimum values for $\left\{\gamma_{k}\right\}$ are calculated using an iterative algorithm in Algorithm 2, which is based on the ellipsoid method [19]. The subgradients required to update them are

$$
d_{k}=\operatorname{tr}\left(\sum_{i=1}^{R} \mathbf{B}_{k} \mathbf{V}_{i} \mathbf{T}_{i} \mathbf{V}_{i}^{H}\right)-P_{k}^{\max } \quad k=1, \ldots, B
$$

Finally, notice that once we have the optimal solution for $\left\{\gamma_{k}\right\}$, we can compute the optimal solution for $\left\{\varphi_{i}\right\}$ following (36), and hence we can directly obtain the optimal solution to the problems $\left(P_{\mathrm{DP}}\right)$ and $\left(P_{1}\right)$. In this respect, an effective algorithm is proposed in Algorithm 2 to solve the resource allocation on the BS-RS link subject to per-BS power constraints, taking into account the MCS constraints and the constraints imposed by the RS-UE link. In the algorithm, $\left\{\gamma_{k}\right\}$ are updated following the ellipsoid method [19], and for each set of $\left\{\gamma_{k}\right\}$, the optimal solution for $\mathbf{T}_{i}{ }^{\prime}$ s, $\left\{r_{1 i}\right\}$ and $\varphi_{i}^{*}$ are computed following (34) and (36).

\section{Competing interests}

The authors declare that they have no competing interests.

\section{Acknowledgments}

This study was done in the framework of the projects TROPIC FP7 ICT-2011 -8-318784 and COST Action IC0902, funded by the European Community. Also, by the Spanish Science and Technology Commissions and EC FEDER funds through projects: TEC2010-19171/TCM and CONSOLIDER INGENIO
CSD2008-00010 COMONSENS, and by project 2009SGR1236 (AGAUR) of the Catalan Administration.

Received: 9 March 2012 Accepted: 21 December 2012

Published: 23 January 2013

\section{References}

1. P Bhat, S Nagata, L Campoy, I Berberana, T Derham, G Liu, X Shen, P Zong, J Yang, LTE-advanced: an operator perspective. IEEE Commun. Mag. 50(2), 104-114 (2012)

2. EEE 802.16 Broadband Wireless Access Group, IEEE 802,16 m system requirements, IEEE $802.16 \mathrm{~m}-07 / 002 \mathrm{r}$. (2007)

3. 3GPP TR 36.912, LTE, Feasibility study for Further Advancements for E-ULTRA (LTE-Advanced). (2010). v9.3.0 Release9

4. Artist4G Consortium, Advanced Relay Technical Proposals, Deliverable D.3.2, ICT-247223 ARTIST4G, 2011

5. C Hoymann, W Chen, J Montojo, A Golitschek, C Koutsimanis, X Shen, Relaying operation in 3GPP LTE: challenges and solutions. IEEE Commun. Mag. 50(2), 156-162 (2012)

6. A Host-Madsen, J Zhang, Capacity bounds and power allocation for wireless relay channels. IEEE Trans. Inf. Theory 51(6), 2020-2040 (2005)

7. A Agustin, J Vidal, O Munoz, Protocols and resource allocation for the twoway relay channel with half-duplex terminals, in IEEE International Conference on Communications (ICC) (, Dresden, 14-18 June 2009), pp. 1-5

8. RS Blum, MIMO capacity with interference. IEEE J. Sel. Areas Commun 21(5), 793-801 (2003)

9. H Takahashi, Y Ofuji, S Fukumoto, S Abeta, T Nakamura, Field experimental results on E-UTRA downlink throughput using polarization diversity antennas, in IEEE Vehicular Technology Conference (VTC) (, Barcelona, 26-29 April 2009), pp. 1-6

10. MK Karakayali, GJ Foschini, RA Valenzuela, Network coordination for spectrally efficient communications in cellular systems. IEEE Wireless Commun. 13(4), 56-61 (2006)

11. D Lee, H Seo, B Clerckx, E Hardouin, D Mazzarese, S Nagata, K Sayana, Coordinated multipoint transmission and reception in LTE-advanced: deployment scenarios and operational challenges. IEEE Commun. Mag. 50 (2), 148-155 (2012)

12. R Zhang, Cooperative multi-cell block diagonalization with per-base-station power constraints. IEEE J. Sel. Areas Commun 28(9), 1435-1445 (2010)

13. J Vidal, A Agustin, S Lagen, E Valera, O Muñoz, A Garcia, M Sanchez, Network-MIMO backhauling for QoS-constrained relay transmission, in IEEE International Conference on Acoustics, Speech and Signal Processing (ICASSP) (, Prague, 2227 -May 2011), pp. 3332-3335

14. A Agustin, J Vidal, S Lagen, E Valera, Network MIMO for downlink in-band relay transmission with relaying phases of fixed duration, in 19th European Conference on Signal Processing (EUSIPCO) (, Barcelona, 29 August - 2 September 2011), pp. 1-5

15. J Zhang, R Chen, J Andrews, A Ghosh RW, Heath, networked MIMO with clustered linear precoding. IEEE Trans. Wirel. Commun 8(4), 1910-1921 (2009)

16. G Kramer, M Gastpar, P Gupta, Cooperative strategies and capacity theorems for relay networks. IEEE Trans. Inf. Theory 51(9), 3037-3063 (2005)

17. QH Spencer, AL Swindlehurst, M Haardt, Zero-forcing methods for downlink spatial multiplexing in multiuser MIMO channels. IEEE Trans. Signal Process. 52(2), 461-471 (2004)

18. S Shi, M Schubert, N Vucic, H Boche, MMSE optimization with per-base -station power constraints for network MIMO systems, in IEEE International Conference on Communications (ICC) (, Beijing, 1923 -May 2008), pp. $4106-4110$

19. S Boyd, L Vandenberghe, Convex Optimization (Cambridge University Press, Cambridge, MA, 2004) 
20. W Yu, R Lui, Dual methods for nonconvex spectrum optimization of multicarrier systems. IEEE Trans. Commun. 54(7), 1310-1322 (2006)

21. WINNER II consortium, Channel Models Part II, Radio Channel Measurements and Analysis Results, 2007. Deliverable 1.1.2, IST-4-027756 WINNER II

22. Femto Forum, Interference management in OFDMA femtocells. (2010)

23. T Cover, J Thomas, Elements of Information Theory (Wiley, New York, 1991)

doi:10.1186/1687-1499-2013-13

Cite this article as: Lagen et al:: Network-MIMO for downlink in-band relay transmissions. EURASIP Journal on Wireless Communications and Networking 2013 2013:13.

Submit your manuscript to a SpringerOpen ${ }^{\circ}$ journal and benefit from:

- Convenient online submission

- Rigorous peer review

- Immediate publication on acceptance

- Open access: articles freely available online

- High visibility within the field

- Retaining the copyright to your article

Submit your next manuscript at $>$ springeropen.com 